\title{
Correction to: Various Shapes of Cultural Biosemiotics
}

\author{
Jonathan Hope ${ }^{1}$
}

Published online: 10 June 2018

(C) Springer Science+Business Media B.V., part of Springer Nature 2018

\section{Correction to: Biosemiotics 201710 (3): 397-411 https://doi.org/10.1007/s12304-017-9307-6}

In the original published article, in discussing Paul Cobley's work, I made an unclear statement and one that was actually false. I mentioned that Cobley is critical of "otherness" as it has been explored in sex and gender studies. More precisely, I should have stated that Cobley is critical of the way otherness has sometimes been cast; plus, sex and gender issues are in fact one of many examples Cobley deals with. I also inadvertently made the mistake of linking parenting to heteronormativity, and for this I apologize as parenting is not an essentially heterosexual feature. In no way was my critique of Cobley an implicit accusation of homophobia, but a sincere attempt to engage with the text and draw out conclusions of what was being said concerning the links between biosemiotics and human cultural studies. Finally, I wish to apologize to Paul Cobley for the effects of this unfortunate interpretation, and thank him, and the editors of the journal, for collaborating in drafting this erratum.

The correct wording (page 401, second paragraph) is as follows, but does not affect the conclusion of the article:

First, at the end of his fourth chapter, "The natural subject" Cobley writes:

what is 'other' in biosemiotics is conceived in a more far-reaching fashion than in other theories of subjectivity. Customarily, the other has been someone - or, occasionally, some thing - else. It is often another gender, another sexuality, another ethnicity,

The online version of the original article can be found at https://doi.org/10.1007/s12304-017-9307-6

Jonathan Hope

hope.jonathan@uqam.ca

1 Université du Québec à Montréal, Montréal, QC, Canada 
another nationality, another culture in general. In posthumanism, the other has often been a non-human animal, a machine or an organic entity augmented by prostheses such as a cyborg. Invariably, the other is considered in such formulations in order to demonstrate the 'undecideability' that arises in attempts to pin down the nature of the human and subjectivity. As has been seen, biosemiotics is not prone to such squeamish prevarication; it is committed to revealing what it is to be human and what it is to be a natural subject. (2016: 58).

Cobley presents here a critical interpretation of the way that the parameters of otherness are sometimes drawn. The scope of otherness explored in the broad cultural studies - including (but probably not limited to) issues relating to sex and gender, ethnicity, nationality - and the more recent developments in posthumanist theory - nonhumans, machines, cyborgs - may be not as essential, so to speak, as the plethora of biotic relations that make up living systems. Yet, if the objective is for us, biosemioticians, to cast bridges with other communities, it might be interesting to see what we can learn from them and see how their insights could transform our research. For example, we could consider an alternative to the ending of Cobley's fifth chapter, "Ethics cannot be voluntary" where he suggests that parenting is the proper model of semiosis.

Parenting necessitates care without the kind of self-interest that Condillac and other humanists have assumed is in ethics. It is a semiosis that, for humans, contains experience of pleasure, pain, sadness and happiness in begetting further semioses of experience of pleasure, pain, sadness and happiness. It answers the call of the other. More than any of these, it is central to reproduction of the Umwelten of all species. Sometimes it is called love. (2016: 73).

Since we are dealing with metaphors, couldn't we say that friendship, a dialectical and sometimes chosen solidarity, is an appropriate model for certain relationships in nature, such as symbiosis? On this particular issue of care, gender and queer theory within ecological debates (Mortimer-Sandilands and Erickson 2010) has proven itself to be not only relevant but also absolutely necessary. Equally relevant is María Puig de la Bellacasa's recent research on soil care (2015), intersecting science and nature. Care goes in all directions, it circulates. A caring relationship is precisely that: a relationship, where no body, nothing, no person, no subject, no interpretant, not even human, is endowed with any presidential status.

This should replace the following:

First, there is a certain political/ideological ambiguity in Cobley's book: this ambiguity can actually be stimulating and, in any case, it is unavoidable in that living processes cannot be partitioned into simplistic models of political behaviour. But sometimes ambiguity can be tendentious. For instance, Cobley ends his fourth chapter, "The Natural Subject" with a curious remark where he seems to lambaste the whole field of gender and queer studies, an academic trend of sexual otherness loosely associated to the left, qualifying its work as "squeamish prevarication" (2016: 58). I think I see what Cobley is getting at: the scope of otherness implied by "highly specialized sexual practices" (Cobley 2016: 59) are minor, so to speak, compared to the plethora of biotic relations that make up living systems. However, I am not sure that his harsh focus on gender issues is an effective way to build bridges with other communities - let alone if 
it adequately grasps the full potential of those issues (something I do not myself claim to master). This tangent culminates, at the end of Cobley's fifth chapter, trenchantly titled "Ethics cannot be voluntary", with a heteronormative claim according to which parental love is the proper model of semiosis. However, we could say that friendship, a democratic, dialectical and sometimes chosen solidarity, is probably a more appropriate model for certain relationships in nature, for example in the case of symbiosis. On this particular issue of care, gender and queer theory within ecological debates (MortimerSandilands and Erickson 2010) has proven itself to be not only relevant but also absolutely necessary. Equally relevant is María Puig de la Bellacasa's recent research on soil care (2015), intersecting science and nature. Care goes in all directions, it circulates. A caring relationship is precisely that, a relationship; if we start attributing roles to agents, we risk objectifying them at which point the relationship becomes secondary. Therefore, we should question the ideological assumptions behind Cobley's proposal to endow humans with a special "presidential" status. 